CLINICAL ARTICLE

\title{
Association of maternal sleep practices with pre-eclampsia, low birth weight, and stillbirth among Ghanaian women
}

\author{
Jocelynn T. Owusu a,b, Frank J. Anderson a,c , Jerry Coleman ${ }^{\mathrm{d}}$, Samuel Oppong ${ }^{\mathrm{d}}$, Joseph D. Seffah ${ }^{\mathrm{d}}$, \\ Alfred Aikins ${ }^{\mathrm{d}}$, Louise M. O'Brien ${ }^{\mathrm{b}, \mathrm{e}, *}$ \\ a School of Public Health, University of Michigan, Ann Arbor, USA \\ b Sleep Disorders Center, Department of Neurology, University of Michigan, Ann Arbor, USA \\ ' Department of Obstetrics and Gynecology, University of Michigan, Ann Arbor, USA \\ d Department of Obstetrics and Gynecology, Korle Bu Teaching Hospital, Accra, Ghana \\ e Department of Oral and Maxillofacial Surgery, University of Michigan, Ann Arbor, USA
}

\section{A R T I C L E I N F O}

\section{Article history:}

Received 7 November 2012

Received in revised form 10 January 2013

Accepted 15 February 2013

\section{Keywords:}

Africa

Low birth weight

Pre-eclampsia

Sleep position

Sleep quality

Snoring

Stillbirth

\begin{abstract}
A B S T R A C T
Objective: To assess sleep practices, and investigate their relationship with maternal and fetal outcomes, among pregnant Ghanaian women. Methods: In a cross-sectional study conducted at Korle Bu Teaching Hospital, Accra, Ghana, between June and July 2011, postpartum women were interviewed within 48 hours of delivery about sleep quality and practices during pregnancy. Interviews were coupled with a systematic review of participants' medical charts for key outcomes including maternal hypertension, pre-eclampsia, premature delivery, low birth weight, and stillbirth. Results: Most women reported poor sleep quality during pregnancy. Snoring during pregnancy was independently associated with pre-eclampsia (odds ratio [OR], 3.5; 95\% confidence interval $[\mathrm{CI}], 1.4-8.5 ; P=0.007)$. The newborns of women who reported supine sleep during pregnancy were at increased risk of low birth weight $(\mathrm{OR}, 5.0 ; 95 \% \mathrm{CI}, 1.2-20.2 ; P=0.025)$ and stillbirth $(\mathrm{OR}, 8.0 ; 95 \% \mathrm{CI}$, $1.5-43.2 ; P=0.016$ ). Low birth weight was found to mediate the relationship between supine sleep and stillbirth. Conclusion: The present findings in an African population demonstrate that maternal sleep, a modifiable risk factor, has a significant role in pre-eclampsia, low birth weight, and subsequently stillbirth.
\end{abstract}

(c) 2013 International Federation of Gynecology and Obstetrics. Published by Elsevier Ireland Ltd. All rights reserved.

\section{Introduction}

Despite the Safe Motherhood Initiative launched in 1987 [1] and the Millennium Development Goals-a UN declaration to reduce maternal and child morbidity and mortality by 2015 [2]-maternal mortality remains high in low-income countries. Sub-Saharan Africa has the highest maternal and perinatal mortality rate in the world [3]. In Ghana, the current maternal mortality rate is 350 per 100000 live births, compared with 21 per 100000 live births in the USA [3]. In Africa, hypertensive disorders of pregnancy are a leading cause of mortality [4-6].

Located in Accra, Korle Bu Teaching Hospital is the largest hospital in Ghana and is associated with the University of Ghana Medical School. More than 12000 women deliver at Korle Bu each year, including both self-referrals from the Greater Accra region and tertiary referrals. Between 1995 and 2002, there were 108 maternal and/or perinatal deaths among 93622 deliveries in Korle Bu Teaching Hospital, and 22 women died before delivery [4]. The leading cause of death was the presence of a hypertensive disorder, and the majority (66\%) of perinatal

\footnotetext{
* Corresponding author at: Michael Aldrich Sleep Disorders Laboratory, C728 Med Inn, Box 5845, 1500 East Medical Center Drive, Ann Arbor, MI, 48109-0845 USA. Tel.: + 1734 647 9064; fax: + 17346479065 .

E-mail address: louiseo@med.umich.edu (L.M. O'Brien).
}

deaths were stillbirths. Sub-Saharan Africa has the highest rate of stillbirth [7], and little progress has been made in mortality reduction. Addressing these disparities presents a major challenge to public health.

Epidemiologic studies from high-resource countries show that poor sleep, particularly sleep-disordered breathing-a spectrum of sleeprelated breathing conditions including snoring and obstructive sleep apnea-is causally related to hypertension [8] via mechanisms that include autonomic dysfunction, endothelial dysfunction, and inflammation [9]. Emerging data also demonstrate that sleep-disordered breathing during pregnancy is common, affecting up to one-third of women in their last trimester [10,11]; it has an independent association with gestational hypertension and pre-eclampsia [10-13] and possibly intrauterine growth restriction [12-14]. Furthermore, poor sleep quality, short sleep duration, and sleep position might all be involved in adverse pregnancy outcomes such as cesarean delivery [15], preterm birth [16], and stillbirth [17].

Although sleep disruption in pregnancy may jeopardize maternal blood pressure control and contribute to adverse pregnancy outcomes, reports of sleep disruption are rare in Ghana and non-existent among the pregnant population. Therefore, the aim of the present study was to investigate the prevalence of sleep disruption and sleep practices among Ghanaian women and to investigate its association with maternal and neonatal outcomes. 


\section{Materials and methods}

In an interview-based study, postpartum Ghanaian women were recruited from maternity wards at Korle Bu Teaching Hospital Accra, Ghana, between June 1 and July 31, 2011. Written informed consent was obtained from all participants. The study was approved by Institutional Review Boards at the University of Michigan and the University of Ghana.

The inclusion criteria were delivery within 48 hours of recruitment and a gestation of at least 28 weeks. There were no other exclusion criteria. Both consent and the interview were verbally administered in the primary language of the participant (English, Twi, or Ga) by a research assistant who was blind to the delivery outcome. Because the study formed part of a student summer project, recruitment was limited by the availability of the research assistant.

There are no validated scales for assessing sleep in sub-Saharan Africa. As a result, the questionnaires were based on those that have been validated in high-income countries. Participants were asked 2 questions about their regular sleeping location: "is your sleeping area (bed) uncomfortable (e.g. hard mattress or floor, too many or not enough blankets)?" and "is your sleeping room uncomfortable (e.g. too hot, bright, stuffy, or noisy)?"

Snoring, as a key symptom of sleep-disordered breathing, was considered to be present if there was a positive response to the question "do you snore loudly?" regardless of whether the response was obtained by self-report or by the comments of a partner. Witnessed episodes of apnea were considered to be present if there was a positive response to the question "has anyone observed you stop breathing during your sleep?"

Sleep quality was measured via a modified version of the sleep quality subscale of the General Sleep Disturbance Scale [18] (GSDS; Supplementary Material S1). This subscale consists of 7 items, and women were asked whether or not they had experienced each item at least 3 nights per week. This dichotomy, although not part of the original GSDS validation, was used because of language difficulties. Women were considered to have poor sleep quality if they positively endorsed 3 or more items. Similarly, daytime sleepiness was measured using a dichotomized version of the sleepiness subscale of the GSDS (Supplementary Material S2), and women were considered to have daytime sleepiness if they positively endorsed 3 or more items (the last item was reverse-scored). The proportion of women with poor sleep quality (or daytime sleepiness) was then calculated.

Usual bedtime and wake time were obtained and used to calculate average sleep duration. Short sleep was defined as 6 hours per night or less, and long sleep was defined as 10 hours per night or more. Women were also queried about their typical sleep position during pregnancy ("while pregnant, what is your most common sleep position?"). Women could provide an open-ended response, which was then coded into "side", "back", "back and side," or "don't know". Supine sleep was considered present if the participant reported "back," or "back and side" sleep. Women were also asked whether they used a treated mosquito net at night. Mosquito nets were considered an important covariate because they are recommended for malaria control and reduce nocturnal mosquito bites, which might potentially improve sleep quality.

Medical records were accessed to obtain key variables including age, gravidity, parity, blood pressure readings, and diagnoses of gestational hypertension or pre-eclampsia. Hypertension was defined as at least 2 blood pressure readings of $140 / 90 \mathrm{~mm} \mathrm{Hg}$ or higher. Newborn outcomes included mode of delivery, gestational age, birth weight, Apgar scores, neonatal intensive care unit (NICU) admission, and stillbirth. Neonates were defined as premature if delivery occurred before 37 weeks of gestation, and as low birth weight if they weighed $2500 \mathrm{~g}$ or less [19].

All data were double-entered to ensure accuracy and were analyzed with SPSS version 19.0 (IBM, Armonk, NY, USA). Histograms, box plots, and descriptive methods were used to check data for errors and outliers. The population attributable risk percent (PAR\%), which represents the proportion of disease (pre-eclampsia, low birth weight, or stillbirth) among the total population that would not have occurred in the absence of exposure (snoring or sleep position), assuming that the exposure contributed in a causal manner to the disease, was calculated as follows:

$\mathrm{PAR} \%=$ incidence $_{\text {total population }}-$ incidence $_{\text {unexposed }} /$ incidence $_{\text {total population }}$

Between-group comparisons of continuous variables were done with a $t$ test. Dichotomized variables were compared with a $\chi^{2}$ test. The Pearson correlation coefficient was calculated to assess relationships among continuous variables. Logistic regression was used to determine associations between maternal sleep and pregnancy outcomes, after adjusting for relevant covariates. The odds ratio (OR) and 95\% confidence interval $(\mathrm{CI})$ were calculated. A $P$ value 0.05 or less was considered to be statistically significant.

\section{Results}

During the study period, 234 women were invited to participate and 232 (99\%) provided consent. Pregnancy outcome data were available for 220 (94.8\%) participants; there was no or limited documentation for the remaining women. As a result, the sample size for all subsequent analyses was 220 women. There were no demographic differences between those with and those without complete outcome data (data not shown).

Overall, $10.9 \%$ of women had hypertension and $11.8 \%$ had preeclampsia (Table 1). One woman had eclampsia. The mean \pm SD body mass index (BMI, calculated as weight in kilograms divided by the square of height in meters) was $24.3 \pm 4.1$, but because height and weight data were available for only $47.7 \%$ of the participants, BMI was not included in the data analysis.

Overall, $24 \%$ of women reported snoring and a large proportion (42.7\%) reported either long or short sleep duration (Table 2). The majority of women reported apparent exclusive side sleep. Because the number of women who reported exclusive supine sleep was small

Table 1

Demographic data of the study participants $(n=220){ }^{a}$

\begin{tabular}{ll}
\hline Characteristic & Value \\
\hline Age & $29.0 \pm 5.7$ \\
$14-19 \mathrm{y}$ & $10(4.5)$ \\
$20-29 \mathrm{y}$ & $108(49.1)$ \\
$30-39 \mathrm{y}$ & $96(43.6)$ \\
$\geq 40 \mathrm{y}$ & $6(2.7)$ \\
Gestational age at delivery & $38.3 \pm 2.6$ \\
$22-26$ wk & $3(1.4)$ \\
$27-31 \mathrm{wk}$ & $3(1.4)$ \\
$32-36$ wk & $35(15.9)$ \\
$37-41 \mathrm{wk}$ & $171(77.7)$ \\
$\geq 42$ wk & $8(3.6)$ \\
Gravidity & \\
1 & $47(21.3)$ \\
2 & $59(26.9)$ \\
3 & $48(21.8)$ \\
4 & $34(15.3)$ \\
$\geq 5$ & $32(14.7)$ \\
Parity & \\
0 & $71(32.3)$ \\
1 & $62(28.2)$ \\
2 & $51(23.2)$ \\
3 & $22(10.0)$ \\
4 & $9(4.1)$ \\
Hypertension (excluding pre-eclampsia) & $5(2.3)$ \\
Pre-eclampsia & $24(10.9)$ \\
\hline
\end{tabular}

a Values are given as mean \pm SD or number (percentage). 
Table 2

Sleep characteristics of the study participants $(n=220){ }^{\text {a }}$

\begin{tabular}{ll}
\hline Characteristic & Value \\
\hline Sleep duration per night & $8.3 \pm 2.2$ \\
$\leq 6 \mathrm{~h}$ & $37(16.8)$ \\
$\geq 10 \mathrm{~h}$ & $57(25.9)$ \\
Snoring & $54(24.0)$ \\
Poor sleep quality & $190(86.4)$ \\
Daytime sleepiness & $115(52.3)$ \\
Supine sleep & $21(9.7)$ \\
Uncomfortable room & $61(27.7)$ \\
Uncomfortable bed & $44(20.0)$ \\
Slept near a light source & $85(38.6)$ \\
Used a treated mosquito net & $49(22.3)$ \\
\hline a Values are given as mean \pm SD or number (percentage).
\end{tabular}

$(\mathrm{n}=4)$, those who reported "back and side sleep" were included as supine sleepers for the purpose of analysis. There were small but significant negative correlations between sleep duration and maternal age $(r=-0.34 ; P<0.001)$ and parity $(r=-0.14 ; P=0.038)$. However, there were no associations between sleep practices and sleep environment, including use of a mosquito net (Table 3 ).

The sleep characteristics of snoring women did not differ from those of non-snorers (Table 4). Snoring women, however, were more likely to have pre-eclampsia than were non-snorers $(22.6 \% \mathrm{vs}$ $7.8 \% ; P=0.0065$ ).

In a logistic regression model controlling for maternal age, parity, and gestational age at delivery, snoring was independently associated with pre-eclampsia ( $\mathrm{OR}, 3.5 ; 95 \% \mathrm{CI}, 1.4-8.5, P=0.007)$. If snoring has a causative role in pre-eclampsia, the PAR\% value suggests that $34 \%$ of cases of pre-eclampsia may be ameliorated by treating snoring.

Snoring, sleep quality, and sleep duration were not associated with the delivery outcomes ( prematurity, cesarean delivery, low birth weight, NICU admission, or stillbirth). Women who reported supine sleep were, however, at increased risk of low birth weight (36.8\% vs $10.7 \%$; $P=$ $0.004)$, NICU admission (36.8\% vs $15.2 \% ; P=0.04)$, and stillbirth $(15.8 \%$ vs $3.0 \% ; P=0.04$ ) (Table 5 ).

In a logistic regression that controlled for the covariates maternal age, gestational age, parity, and the presence of pre-eclampsia, supine sleep was independently associated with low birth weight (OR, 5.0; $95 \%$, CI 1.2-20.2; $P=0.025)$. Similarly, in a separate logistic regression model that controlled for the same covariates, supine sleep was independently associated with stillbirth (OR, 8.0; 95\% CI, 1.5-43.2; $P=0.016$ ). When low birth weight was added to the latter model, the odds ratio for the relationship between supine sleep and stillbirth was almost eliminated (OR, 4.9; 95\% CI, 0.80-31.4; $P=0.09$ ). This demonstrates that low birth weight mediates the effects of supine sleep on stillbirth. If supine sleep has a causative role in low birth weight and subsequently stillbirth, the PAR\% suggests that up to $17 \%$ of low birth weight, and consequently $26 \%$ of stillbirths, might be prevented by changing maternal sleep position.
Table 4

Sleep characteristics and pregnancy outcomes among snoring and non-snoring women. ${ }^{\mathrm{a}}$

\begin{tabular}{llll}
\hline Characteristic & $\begin{array}{l}\text { Snoring } \\
(\mathrm{n}=53)\end{array}$ & $\begin{array}{l}\text { Non-Snoring } \\
(\mathrm{n}=167)\end{array}$ & P value \\
\hline Age, y & $29.1 \pm 5.5$ & $28.8 \pm 5.8$ & \\
Parity & $1.4 \pm 1.5$ & $1.3 \pm 1.3$ & \\
Sleep duration, h & $8.1 \pm 2.4$ & $8.4 \pm 2.2$ & \\
Short sleep duration & $11(20.9)$ & $27(16.2)$ & \\
Long sleep duration & $13(24.5)$ & $46(27.5)$ & \multirow{2}{*}{0.05} \\
Witnessed apneas & $7(13.2)$ & $8(4.8)$ & \\
Poor sleep quality & $45(84.9)$ & $144(86.2)$ & \\
Excessive daytime sleepiness & $28(52.8)$ & $87(52.1)$ & \\
Supine sleep & $5(9.4)$ & $14(8.4)$ & \\
Hypertension (excluding pre-eclampsia) & $4(7.7)$ & $21(12.6)$ & \\
Pre-eclampsia & $12(22.6)$ & $13(7.8)$ & \\
Cesarean delivery & $19(35.8)$ & $42(25.1)$ & \\
Low birth weight & $6(11.3)$ & $21(12.6)$ & \\
NICU admission & $12(22.6)$ & $25(15.0)$ & \\
Stillbirth & $2(3.8)$ & $7(4.2)$ & \\
\hline
\end{tabular}

Abbreviation: NICU, neonatal intensive care unit.

a Values are given as mean \pm SD or number (percentage) unless stated otherwise.

\section{Discussion}

The present study has shown that maternal snoring and sleep position during pregnancy confer a significant risk on maternal and perinatal outcomes in a low-resource country. Pre-eclampsia, low birth weight, and stillbirth are major contributors to the high perinatal mortality rate in Ghana, and the present findings suggest that maternal sleep may have a role.

Increasing evidence in high-income countries indicates that maternal sleep affects maternal blood pressure [11,12,20]. A large prospective study previously showed that pregnancy-onset snoring was independently associated with gestational hypertension and pre-eclampsia [11]. From the results of the present cross-sectional study, it is not possible to determine whether snoring increases the risk of pre-eclampsia or whether pre-eclampsia increases the risk of snoring. The relationship is likely to be bidirectional.

Epidemiologic studies among non-pregnant adults have demonstrated that snoring is predictive of hypertension [21] owing to endothelial dysfunction, inflammation, oxidative stress, and increased sympathetic activity [9]-pathways that are also triggered in pre-eclampsia. Although there is clear overlap in the biologic pathways, pre-eclampsia is thought to originate in early gestation and manifest later in pregnancy; however, it is likely that snoring exacerbates the underlying physiology and accelerates the development of pre-eclampsia [11]. Conversely, women with pre-eclampsia are also at high risk of snoring [22]. These findings suggest that the relationship between snoring and pre-eclampsia may be bidirectional.

Notably, supine sleep during pregnancy was found to be associated with stillbirth via a mediating effect of low birth weight. Women in Ghana have one of the highest rates of stillbirth in the world, yet interventions have had little success [7]. The present findings support those of Stacey et al. [17], who found that supine sleep was associated with a 2.5-fold increased risk of stillbirth in New Zealand, although

Table 3

Associations between environment and sleep practice. ${ }^{a}$

\begin{tabular}{|c|c|c|c|c|c|c|c|c|}
\hline & \multicolumn{2}{|c|}{ Uncomfortable room } & \multicolumn{2}{|c|}{ Uncomfortable bed } & \multicolumn{2}{|c|}{ Use of mosquito net } & \multicolumn{2}{|c|}{ Slept near a light source } \\
\hline & Yes & No & Yes & No & Yes & No & Yes & No \\
\hline Sleep duration, h & $8.4 \pm 2.0$ & $8.3 \pm 2.3$ & $8.0 \pm 2.2$ & $8.4 \pm 2.2$ & $8.3 \pm 2.1$ & $8.2 \pm 2.3$ & $8.4 \pm 2.3$ & $8.2 \pm 2.2$ \\
\hline Short sleep & 16.1 & 17.5 & 21.3 & 16.0 & 21.1 & 16.0 & 15.9 & 17.9 \\
\hline Long sleep & 25.8 & 26.6 & 17.0 & 28.8 & 26.4 & 26.6 & 31.8 & 23.1 \\
\hline Poor sleep quality & 88.7 & 85.3 & 87.2 & 85.9 & 86.8 & 86.0 & 87.6 & 85.3 \\
\hline
\end{tabular}

${ }^{\text {a }}$ Values are given as mean $\pm \mathrm{SD}$ or percentage. 
Table 5

Delivery outcomes associated with supine sleep $(n=216){ }^{a}$

\begin{tabular}{llll}
\hline & $\begin{array}{l}\text { Supine sleep } \\
(\mathrm{n}=19)\end{array}$ & $\begin{array}{l}\text { Non-supine sleep } \\
(\mathrm{n}=197)\end{array}$ & $P$ value \\
\hline $\begin{array}{l}\text { Gestational age, wk } \\
\text { Birth weight, g }\end{array}$ & $\begin{array}{l}37.0 \pm 4.3 \\
2830.5 \pm 949.4\end{array}$ & $\begin{array}{l}38.2 \pm 2.7 \\
3073.4 \pm 601.2\end{array}$ & \\
Apgar score & & & \\
1 min & $6.9 \pm 1.5$ & $7.0 \pm 1.0$ & \\
5 min & $8.2 \pm 1.3$ & $8.3 \pm 0.9$ & \\
Premature delivery & $6(31.6)$ & $35(17.8)$ & 0.004 \\
Cesarean delivery & $5(26.3)$ & $57(28.9)$ & 0.04 \\
Low birth weight & $7(36.8)$ & $21(10.7)$ & 0.04 \\
NICU admission & $7(36.8)$ & $30(15.2)$ & \\
Stillbirth & $3(15.8)$ & $6(3.0)$ & \\
\hline
\end{tabular}

Abbreviation: NICU, neonatal intensive care unit.

${ }^{a}$ Values are given as mean \pm SD or number (percentage) unless stated otherwise. Four individuals reported that they did not know their sleep position; thus, the total sample size was 216 .

they did not report birth weight. In their case-control study, women were interviewed within several weeks of delivery; in the present study, women were interviewed within 48 hours of delivery with the researcher blind to delivery outcomes, thereby minimizing biases. Similar to the study of Stacey et al. [17], the present data suggest that (if sleep position is causally related to stillbirth) more than one-quarter of stillbirths might be avoided by altering maternal sleep position.

The present study found an association between supine sleep and low birth weight, which might mediate the relationship between supine sleep and stillbirth. More than 20 million neonates with low birth weight are born annually worldwide, with the highest concentration in Asia and Africa [3]. Efforts to reduce the incidence of low birth weight have not been successful in these regions. The possibility that supine sleep has a part in low birth weight, and subsequently stillbirth, is plausible due to uterine compression on the inferior vena cava, resulting in reduced venous filling and cardiac output [23]. A study in awake pregnant women confirmed that altering maternal position from supine to left lateral significantly increased the ejection fraction, and end-diastolic, end-systolic, and cardiac output [24]. The reduction in cardiovascular parameters, particularly cardiac output, in the supine position might impede utero-placental blood flow and increase the risk of growth restriction and stillbirth. Indeed, small-for-gestational-age is a known contributor to stillbirth [25].

In addition to snoring and supine sleep, poor sleep quality and short sleep duration have been postulated to increase the risk of adverse pregnancy outcomes $[15,16,20]$. The vast majority of Ghanaian women reported poor sleep quality; however, no association with maternal or perinatal outcomes was found. Similarly, there was no association between short sleep and pregnancy outcomes. It is possible that poor sleep in early pregnancy may alter placental vascular remodeling and subsequently increase the risk of adverse outcomes [26]; however, because we did not perform a longitudinal study, we are unable to address this possibility.

Unexpectedly, an uncomfortable sleeping location (e.g. room too hot) or uncomfortable bed/mattress was not associated with poor sleep. It is possible that women were used to their typical sleeping locations and so falsely ascribed their poor sleep to pregnancy. However, the prevalence of poor sleep during pregnancy in the present study is similar to that of pregnant women in high-income countries who typically have a large degree of control over the comfort of their sleep locations [27]. Thus, it seems unlikely that comfort of the sleeping location is involved in the poor sleep observed among the study participants.

The present study has several limitations. The lack of objective measures of sleep, such as actigraphy or polysomnography, might have impeded the ability to fully measure sleep variables; however, such measures are not readily available in low-resource countries and simple, efficient screening questions might assist the identification of women at risk of poor birth outcomes. In addition, women were enrolled within 48 hours of delivery; although recall bias is possible, it was minimized by this approach. It was not possible to perform a longitudinal study in this population because often the first time a woman presents to the hospital is when there is a problem or she is ready to deliver. Furthermore, the use of hypertensive medications was not considered. Although this would not have altered the relationship between snoring and a clinical diagnosis of hypertension, it might have led to an underestimation of the number of women with high blood pressure in the absence of a clinical diagnosis. If some women were misclassified, however, the study findings might be stronger than presented here.

In addition, height and weight were not available for half of the women; because BMI has strong relationships with both snoring and pregnancy outcomes, it is unfortunate that it was not possible to address the role of obesity. However, data on height and weight are not routinely collected in sub-Saharan Africa. Moreover, the CIs for the logistic regression models of low birth weight and stillbirth were wide. This is probably due to the relatively small sample size, particularly of stillbirths, coupled with wide variability. Lastly, it was not possible to investigate left side versus right side or to delineate sleep position on settling versus position on waking. Nevertheless, the present study clearly demonstrates that maternal sleep practices in a population with high perinatal morbidity is worthy of further investigation in a much larger sample size.

Given the present findings, it would be prudent to develop simple intervention trials. There is no standard treatment for snoring; any underlying obstructive sleep apnea is typically treated by continuous positive airway pressure, an approach not feasible in low-resource countries. However, snoring may be improved by non-supine sleep because the airway is more likely to collapse when sleeping supine. Moreover, non-supine sleep might also reduce the incidence of low birth weight and subsequently stillbirth. This is a simple intervention that can be performed in low-resource countries. Although it is not possible to obtain confirmatory evidence of sleep position, maternal report of position at bedtime and at wake time might be sufficient. Simply querying a pregnant woman about her sleep is an approach that can be adopted easily even by untrained personnel.

In summary, the present data suggest that maternal sleep, a modifiable risk factor, has a significant role in pre-eclampsia, low birth weight, and stillbirth among Ghanaian women. As a country with one of the highest morbidity and mortality rates in the world, Ghana urgently requires inexpensive interventions to improve maternal and perinatal morbidities. Encouraging pregnant Ghanaian women to avoid sleeping on their back might be one such intervention.

Supplementary data to this article can be found online at http:// dx.doi.org/10.1016/j.ijgo.2013.01.013.

\section{Acknowledgments}

The project was supported by a grant from the National Center of Minority Health \& Health Disparities at the National Institutes of Health (T37 MD001425-11), and administered by the Center for Human Growth \& Development of the University of Michigan. L.M.O. was supported by a career grant from the National Heart, Lung, and Blood Institute (K23 HL095739) and in part by R21 HL087819 and R21 HL089918.

\section{Conflict of interest}

The authors have no conflicts of interest.

\section{References}

[1] Mahler H. The safe motherhood initiative: a call to action. Lancet 1987;1(8534): 668-70.

[2] Millenium Project. Investing in Development. http://www.unmillenniumproject. org/reports/fullreport.htm. [Published 2006]. 
[3] World Health Organization, UNICEF, UNFPA, The World Bank. Trends in maternal mortality: 1990 to 2010. http://whqlibdoc.who.int/publications/2012/9789241503631_ eng.pdf. Published 2012.

[4] Lassey AT, Obed SA. Trends in concurrent maternal and perinatal deaths at teaching hospital in Ghana: the facts and prevention strategies. J Obstet Gynaecol Can 2004;26(9):799-804.

[5] Zakariah AY, Alexander S, van Roosmalen J, Buekens P, Kwawukume EY, Frimpong P. Reproductive age mortality survey (RAMOS) in Accra, Ghana. Reprod Health 2009;6:7.

[6] Kwawununu FK, Morhe ES, Konney TO. Trends in maternal mortality at Komfo Anokye Teaching Hospital, Kumasi, Ghana, between 1998 and 2007. Int J Gynecol Obstet 2012:117(2):183-4.

[7] Cousens S, Blencowe H, Stanton C, Chou D, Ahmed S, Steinhardt L, et al. National, regional, and worldwide estimates of stillbirth rates in 2009 with trends since 1995: a systematic analysis. Lancet 2011;377(9774):1319-30.

[8] Peppard PE, Young T, Palta M, Skatrud J. Prospective study of the association between sleep-disordered breathing and hypertension. N Engl J Med 2000;342(19):1378-84.

[9] Lavie L. Oxidative stress inflammation and endothelial dysfunction in obstructive sleep apnea. Front Biosci (Elite Ed) 2012;4:1391-403.

[10] Bourjeily G, Raker CA, Chalhoub M, Miller MA. Pregnancy and fetal outcomes of symptoms of sleep-disordered breathing. Eur Respir J 2010;36(4):849-55.

[11] O'Brien LM, Bullough AS, Owusu JT, Tremblay KA, Brincat CA, Chames MC, et al. Pregnancy-onset habitual snoring, gestational hypertension, and preeclampsia: prospective cohort study. Am J Obstet Gynecol 2012;207(6):487.e1-9.

[12] Franklin KA, Holmgren PA, Jönsson F, Poromaa N, Stenlund H, Svanborg E. Snoring, pregnancy-induced hypertension, and growth retardation of the fetus. Chest 2000;117(1):137-41.

[13] Louis JM, Auckley D, Sokol RJ, Mercer BM. Maternal and neonatal morbidities associated with obstructive sleep apnea complicating pregnancy. Am J Obstet Gynecol 2010;202(3):261.e1-5.

[14] Micheli K, Komninos I, Bagkeris E, Roumeliotaki T, Koutis A, Kogevinas M, et al Sleep patterns in late pregnancy and risk of preterm birth and fetal growth restriction. Epidemiology 2011;22(5):738-44.

[15] Lee KA, Gay CL. Sleep in late pregnancy predicts length of labor and type of delivery. Am J Obstet Gynecol 2004;191(6):2041-6.
[16] Okun ML, Schetter CD, Glynn LM. Poor sleep quality is associated with preterm birth. Sleep 2011;34(11):1493-8.

[17] Stacey T, Thompson JM, Mitchell EA, Ekeroma AJ, Zuccollo JM, McCowan LM. Association between maternal sleep practices and risk of late stillbirth: a case-control study. BMJ 2011;342:d3403.

[18] Lee KA. Self-reported sleep disturbances in employed women. Sleep 1992;15(6): 493-8.

[19] World Health Organization. International Statistical Classification of Diseases and Related Health Problems (Icd-10) in Occupational Health. http://www.who.int/ occupational_health/publications/en/oehicd10.pdf. Published 1999.

[20] Williams MA, Miller RS, Qiu C, Cripe SM, Gelaye B, Enquobahrie D. Associations of early pregnancy sleep duration with trimester-specific blood pressures and hypertensive disorders in pregnancy. Sleep 2010;33(10):1363-71.

[21] Nieto FJ, Young TB, Lind BK, Shahar E, Samet JM, Redline S, et al. Association of sleep-disordered breathing, sleep apnea, and hypertension in a large communitybased study. Sleep Heart Health Study. JAMA 2000;283(14):1829-36.

[22] Izci B, Martin SE, Dundas KC, Liston WA, Calder AA, Douglas NJ. Sleep complaints: snoring and daytime sleepiness in pregnant and pre-eclamptic women. Sleep Med 2005;6(2):163-9.

[23] Robson SC, Hunter S, Boys RJ, Dunlop W. Serial study of factors influencing changes in cardiac output during human pregnancy. Am J Physiol 1989;256(4 Pt 2):H1060-5.

[24] Rossi A Cornette J Johnson MR, Karamermer Y, Springeling T Opic P, et al. Quantitative cardiovascular magnetic resonance in pregnant women: cross-sectional analysis of physiological parameters throughout pregnancy and the impact of the supine position. J Cardiovasc Magn Reson 2011;13:31.

[25] Flenady V, Koopmans L, Middleton P, Frøen JF, Smith GC, Gibbons K, et al. Major risk factors for stillbirth in high-income countries: a systematic review and meta-analysis. Lancet 2011;377(9774):1331-40.

[26] Okun ML, Roberts JM, Marsland AL, Hall M. How disturbed sleep may be a risk factor for adverse pregnancy outcomes. Obstet Gynecol Surv 2009;64(4):273-80.

[27] National Sleep Foundation. Summary of Findings. http://www.sleepfoundation.org/ sites/default/files/Summary_Of_Findings\%20-\%20FINAL.pdf. Published 2007. 